\title{
Estrategia de evaluación para el mejoramiento continuo de cursos de un programa de Artes Visuales en modalidad virtual y a distancia: Estudio de caso
}

\section{Evaluation strategy for the continuous improvement of courses of a Visual Arts program in virtual and distance modality: Case study}

\author{
Raúl Alejandro Martínez Espinosa ${ }^{1}$ \\ https://orcid.org/0000-0003-1601-0361 \\ Tatiana Martínez Santis ${ }^{2}$ \\ https://orcid.org/0000-0002-7954-0709 \\ Daniela Hernández Contreras ${ }^{3}$ \\ https://orcid.org/0000-0002-7869-2575
}

Universidad Nacional, Abierta y a Distancia, Colombia

\section{Cita Recomendada}

Martínez, R. A., Martínez, T. \& Hernández, D. (2018) Estrategia de Evaluación para el mejoramiento continuo de cursos de un programa de Artes Visuales en modalidad virtual y a distancia: Estudio de Caso. Hamut'ay, 5 (2), 64-77.

http://dx.doi.org/10.21503/hamu.v5i2.1621

\section{RESUMEN}

La presente investigación se desarrolló con un diseño metodológico de tipo cualitativo, especifícamente estudio de caso, con técnica de recolección de datos grupo focal en estudiantes activos y docentes del programa de artes visuales de una universidad pública de Colombia con modalidad virtual y a distancia. Su objetivo principal fue desarrollar una estrategia de evaluación del programa académico en artes visuales ofertado en ambientes virtuales de aprendizaje. En el año 2016, inicia el programa de artes visuales con una matrícula de 50 estudiantes activos, para el siguiente ańo el número se incrementa a 278. La población de estudiantes, es heterogénea, entre los 16 a los 45 años y condiciones socio-económicas con presencia en 63 centros de educación abierta y a distancia en Colombia. Los resultados concentrados en tres categorías analíticas, siendo: expectativas frente al programa y su metodología, acompañamiento docente y nuevos recursos didácticos y pedagógicos. Su impacto se refleja en los siguientes hallazgos: a. Establecimiento de un programa académico como objete de estudio; b. Diseño de contenidos teórico-metodológicos con recursos educativos digitales

\footnotetext{
1 Artista Plástico y visual. Publicista de la Universidad Jorge Tadeo Lozano, Especialista en periodismo Universidad de Los Andes y Magíster en Artes Plásticas y visuales de la Universidad Nacional de Colombia. Líder Nacional del programa de Artes Visuales de la Universidad Nacional, Abierta y a Distancia - UNAD.raul.martinez@unad.edu.co

2 Psicóloga, Magister en Comunicación Social de la Universidad del Norte, Colombia. Investigadora, docente de la Maestría en Desarrollo Alternativo y Sostenible, y Líder de Investigación de la Escuela de Ciencias Sociales, Artes y Humanidades de la Universidad Nacional, Abierta y a Distancia - UNAD. tatiana.martinez@unad.edu.co

3 Maestro en Música con énfasis en Arreglos musicales de la Universidad El Bosque, Colombia. Candidata a grado Especialista en Educación Superior a Distancia. Docente y Líder del semillero de Investigación del programa de música de la Escuela de Ciencias Sociales, Artes y Humanidades de la Universidad Nacional Abierta y a Distancia UNAD.daniela.hernandez@unad.edu.co
} 
pertinentes e innovadores, que propician el aprendizaje autónomo y significativo; c. Identificación de expectativas y necesidades de docentes y estudiantes activos como parte del proceso de enseńanza y aprendizaje de las artes, metodologías de investigación y creación de obra; d. Implementación de una ruta metodologíca para la evaluación constante, estableciendo prácticas de gestión académica al interior del programa caracterizadas por la calidad, toma de decisiones basadas en investigación y orientadas a la excelencia académica.

Palabras Clave: Artes, recursos educativos, evaluación, gestión académica, entornos virtuales de aprendizaje.

\section{Abstract}

This research was developed with a qualitative methodological design, specifically a case study, with a focus group data collection technique in active students and teachers of the visual arts program of a public university in Colombia with virtual and distance modality. Its main objective was to develop a strategy of evaluation for the academic program in visual arts offered in virtual learning environments. In 2016, the visual arts program starts with an enrollment of 50 active students, for the following year the number increases to 278 . The student population is heterogeneous, between 16 to 45 years old and socio-economic conditions with presence in 63 open and distance education centers in Colombia. The results are concentrated in three analytical categories: expectations regarding the program and its methodology, teacher support and new didactic and pedagogical resources. Its impact is reflected in the following findings: a. Establishment of an academic program as an object of study; b. Design of theoretical-methodological contents with relevant and innovative digital educational resources, which encourage autonomous and meaningful learning; c. Identification of expectations and needs of teachers and active students as part of the teaching and learning process of the arts, research methodologies and creation of work; d. Implementation of a methodological route for constant evaluation, establishing academic management practices within the program characterized by quality, decision-making based on research and oriented towards academic excellence.

Keywords: Arts, educational resources, evaluation, academic management, virtual learning environments.

\section{INTRODUCCIÓN}

La formación de artistas en la modalidad virtual problematiza no sólo la relación arte y tecnología, sino que también aborda aspectos trascendentales de la vida desde diferentes dimensiones (emocionales, cognitivas, expresivas). En este sentido, indagar en el alcance del "comportamiento estético" como lo señala Barbosa (2017) es relevante para los propósitos de formación e investigación en artes:
"Como herramienta posibilitadora de la expresión, que no se desvincula de la vida ni del esfuerzo humano, el arte convoca a los seres humanos a compartir emociones, sensaciones e ideas. Gracias al impulso colectivo, en el arte se logran dos tipos de cumplimiento de la experiencia: la unificación de componentes de la vida y la unión de experiencias intersubjetivas entre colectivos humanos" (p.4).

La modalidad virtual favorece el acceso a la for- 
Estrategia de evaluación para el mejoramiento continuo de cursos de un programa de Artes Visuales en modalidad virtual y a distancia: Estudio de caso

mación en artes, con cobertura nacional e internacional y bajo una estructura flexible para el desarrollo de los contenidos propuestos. Los rasgos distintivos que se proponen desde el programa en relación con otros programas son los siguientes: la virtualidad de sus contenidos curriculares, el activo uso de las tecnologías de la información y la comunicación (TIC) en la relación docente-estudiante y la investigación mediada por tecnologías para la formación en investigación y la investigación formativa desde metodologías propias de las artes como lo es la investigación-creación y la investigación cualitativa o mixta.

La enseñanza de las artes contrae entonces una estrecha relación entre el docente y los estudiantes mediante el taller presencial, empero, gracias a las TIC, es posible que esta relación se de a través de una mediación virtual. Por tratarse del primer programa en esta modalidad que se oferta en Colombia, es necesario indagar en el aprendizaje significativo en cursos teórico-prácticos, es decir, aquellos cursos que en la tradición de la enseñanza en artes se han desarrollado de manera presencial.

Ante las preguntas jes posible aprender a dibujar de manera virtual? ¿se puede formar artistas en la modalidad virtual? las respuestas no pueden surgir de los prejuicios o el entusiasmo sino de la investigación. Esto supone un ejercicio para la revisión del aprendizaje significativo que realizan los estudiantes del programa. En este orden de ideas se propone la formulación de una herramienta para el mejoramiento continuo en el diseño y componentes didácticos de aprendizaje dentro de la modalidad virtual en cursos del programa de artes, siendo: Dibujo, Fotografía y Diseño básico.

El desarrollo de la estrategia de evaluación permite la constante identificación de estandares de éxito/fracaso de la estrategia pedagogica y didáctica implementada para la apropiación de conceptos teorico-prácticos propios de las artes visuales. Para esto se plantearon cuatro fases: En primer lugar, el diseño metodológico, posteriormente la aplicación de técnicas evaluativas, el análisis de resultados y finalmente, formulación de lineamientos.

La educación en ambientes virtuales surge de la necesidad de abarcar todo tipo de contextos y población que por discapacidad, movilidad, espacio y tiempo no pueden acceder a una educación de manera presencial. Esta modalidad de aprendizaje en entornos virtuales ha venido evolucionando a medida que las mismas tecnologías de la información lo permiten, en este momento el crecimiento de cursos en esta modalidad ha venido aumentando y supliendo espacios donde la educación y proyección de conocimientos no era posible, es por esto que las TIC ofrecen distintas herramientas y posibilidades a los estudiantes para adquirir conocimientos, procesarlos y ejecutarlos. Dentro del ambiente de educación superior y a distancia no solo busca el éxito a través de las tecnologías sino también de las mismas herramientas didácticas ofrecidas dentro del curso. Como lo plantea el Plan Nacional de Tecnologías de la Información y las Comunicaciones 2008-2019 del Ministerio de Educación. El uso de estas tecnologías ha cambiado las costumbres sociales y la forma cómo interactúan las personas. Las TIC han mejorado las oportunidades para grandes grupos de la población tradicionalmente excluidos, con lo cual, se ha aumentado la movilidad dentro de la sociedad. Estas tecnologías han producido además una revolución del aprendizaje, cambiando la forma cómo las personas aprenden y el rol de los alumnos y de los maestros. También se ha hecho más claro que el período del aprendizaje no puede ser un proceso limitado en el tiempo, sino que debe darse a lo largo de la vida. (Ministerio de Comunicaciones, 2008, p. 65).

En relación con lo anterior, se recalca la importancia de estar en constante autoevaluación de los cursos en esta modalidad virtual, puesto que la educación a distancia no solo busca brindar la oportunidad a una población o dar fácil acceso al conocimiento sino certificar un aprendizaje educativo de alta calidad.

"La existencia de medios como instrumentos de comunicación e intercambio no garantiza ni determina una metodología ni un aprendizaje concreto ya que se requiere del proceso de construcción y de socialización donde los medios vienen a contribuir, pero no son decisivos para lograr los aprendizajes. La simple presencia de las tecnologías no garantiza resultados óptimos, toda propuesta de aplicación demanda de la participación activa, creativa y 
crítica de los agentes involucrados, cada uno de ellos son generadores de mensajes y contenidos diversos que al poder intercambiar conocimientos e ideas con otros enriquecen el conocimiento" (Ávila \& Bosco, 2001, p.34).

En ese sentido, ¿Qué impacto y efectividad tiene el proceso de aprendizaje en los estudiantes que participan dentro de los cursos metodológicos de artes visuales ofrecidos por una universidad pública con modalidad virtual y a distancia? ¿Qué herramientas pedagógicas se deben tener en cuenta para el mejoramiento continuo en un curso virtual de artes de alta calidad?

Como asevera Ávila \& Bosco (2001) la práctica educativa no es suficiente con la incorporación de las TIC ya que en sí mismas no tienen un significado y un sentido educativo. Debido a esta claridad, el uso de herramientas tecnológicas y comunicativas deben acompañarse de un modelo pedagógico innovador y creativo que permita una constante transformación para un aprendizaje significativo. Desde esta perspectiva se debe garantizar una educación de alta calidad donde los estudiantes tengan resultados de aprendizaje de manera positiva y efectiva.

La investigación explora sobre los aspectos metodológicos de la enseñanza de las artes en ambientes virtuales desde el abordaje de algunos cursos teórico-práctico desde las categorías analíticas de expectativas frente al programa y su metodología, acompañamiento docente y nuevos recursos didácticos y pedagógicos.

Su objetivo central es desarrollar una estrategia de evaluación para el mejoramiento continuo de cuatro cursos metodológicos del programa de artes visuales en ambientes virtuales de aprendizaje: Dibujo, Fotografía y Diseño básico.

Los programas de artes en Colombia: una mirada desde los procesos de enseńanza- aprendizaje.

\section{Oferta de los programas de artes en Colombia}

En la actualidad la oferta de programas de artes en Colombia es insuficiente, en relación con otras profesiones y áreas del conocimiento. Según el SNIES-Ministerio de Educación Nacional, (2013) existen 37 programas en artes que representan tan sólo el $5 \%$ de la oferta activa de educación superior en Colombia. Es decir que, únicamente 469 programas, de los 9.824 que existen, corresponden a esta área del conocimiento. Las áreas de economía, principalmente los programas de administración de empresas e ingeniería concentran más de la mitad de la oferta de los programas ofertados a nivel nacional, con un 55\% del total (ver figura 1).

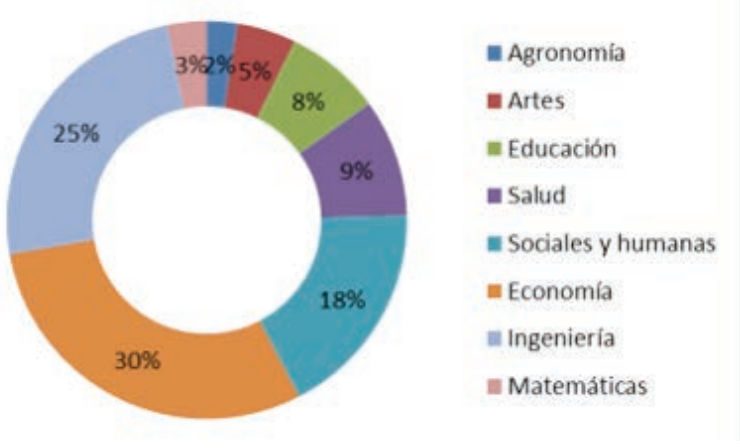

Figura 1

Programas con registro calificado por áreas.

Análisis basado en información del SNIES, Ministerio de Educación Nacional, 2013.

El área de artes está conformada por siete núcleos básicos de conocimiento: Artes plásticas, visuales y afines, Artes Representativas, Historia, Diseño, Música, Publicidad y afines y otras disciplinas sin clasificar; sin embargo, la oferta de artes visuales es significativamente más reducida en comparación con el total de programas que se ofrecen dentro del área. Dentro de los programas de artes, los que ocupan una mayor proporción en número de oferta académica y estudiantes son el de Diseño y Música. De ese $5 \%$ que constituye la oferta a nivel nacional, en el área de artes, se ubican 469 programas que cubren la oferta de los siete núcleos básicos señalados anteriormente. Sólo 69 programas, de esos 469 mencionados, cubren la oferta en artes plásticas, visuales y afines, ya que los demás corresponden al resto de programas que constituyen el área de artes, lo que representa un $19.7 \%$ dentro del área y tan sólo un $0.69 \%$ del total de programas que se ofertan en Colombia, (información recopilada del SNIES-Ministerio de Educación Nacional, 2013 y se puede observar en la Tabla 1). 
Estrategia de evaluación para el mejoramiento continuo de cursos de un programa de Artes Visuales en modalidad virtual y a distancia: Estudio de caso

Tabla 1.

Oferta por áreas del conocimiento. MEN - SACES. Información con corte a Julio de $2013^{*}$ No Incluye los programas de la Universidad Nacional incluye únicamente programas del SENA con Registro Calificado

\begin{tabular}{lcc}
\hline Área & Número de programas & Porcentaje \\
\hline Agronomía & 243 & 2 \\
Artes & 469 & 5 \\
Educación & 769 & 8 \\
Salud & 939 & 9 \\
Sociales y Humanas & 1742 & 18 \\
Economía & 2936 & 30 \\
Ingeniería & 2413 & 25 \\
Matemáticas & 313 & 3 \\
\hline Muy en desacuerdo & 9824 & 100 \\
\hline
\end{tabular}

Análisis basado en información del SNIES, Ministerio de Educación Nacional, 2013

La enseñanza de las artes a través de ambientes virtuales de aprendizaje

En el desarrollo y evolución de las tecnologías de la información se ha buscado crear espacios y entornos en modalidad virtual con el fin de que la educación sea de fácil acceso para todos. Así mismo, se busca que se crean distintos espacios con nuevas oportunidades de conocimiento y formación de educación superior en un ambiente de aprendizaje virtual (Ávila \& Bosco, 2001).

Cuando nos referimos a un ambiente virtual de aprendizaje, se contextualiza como el uso de un software o plataforma, cuyo fin es transmitir información para la construcción de nuevo conocimiento. Un entorno virtual de aprendizaje se caracteriza por el uso de una interface visual intuitiva, donde se hace uso de módulos para la mediación paso a paso de la información, para ello hay herramientas como calendarios, agendas, gestión al estudiante, evaluación y seguimiento, que permitan un real aprendizaje significativo; además de dichas herramientas, hay unos actores que permiten dicha mediación y es un administrador, tutor y estudiante. Un ambiente virtual de aprendizaje debe permitir una comunicación entre el docente y el estudiante para el desarrollo de habilididades y mediación del proceso educativo, además de tener una capacidad de funcionamiento con grandes y pequeños grupos sin afectar la usabilidad de los recursos dispuestos. (Belloch, 2012; Zapata-Ros, 2015).

Según Ávila \& Bosco (2001) se conciben los ambientes de aprendizaje como no suscritos a la educación formal, ni tampoco a una modalidad educativa particular, por el contrario, se tratan de aquellos espacios en los cuales se recrean las condiciones para que el individuo apropie nuevos conocimientos, nuevas experiencias, nuevos elementos que le generen procesos de análisis, reflexión y apropiación sin la obligatoriedad de la presencialidad en escenarios in situ. Desde esta perspectiva, se entiende que la educación a distancia se da pensando en la necesidad que surge en los distintos espacios por tener un aprendizaje y conocimiento específico de distintas áreas, pero que por los espacios y entornos de las mismas personas no es posible tener acceso de manera presencial a estas herramientas educativas. Es así donde las tecnologías de la información (TIC) han jugado un papel determinante en la generación de herramientas y posibilidades de una educación de alta calidad en modalidad virtual y a distancia.

En Colombia el aprendizaje en modalidad virtual y a distancia, ha venido evolucionando en tres momentos, a saber: En primera instancia la interacción de parte del estudiante con el docente, era muy limitada y la mayoría de información era enviada por correo, es aquí donde las TIC han jugado un papel indispensable en esta modalidad, fomentando en un segundo momento un espacio más cercano entre el estudiante y el tutor por medio de espacios radiales y en el ciberespacio al intercambiar información. En su total evolución se llega a un tercer momento; donde el docente tiene un contacto totalmente cercano con el estudiante, gracias a las tecnologías que ofrecen comunicación continua y a las redes que permiten acceder a los procesos de aprendizaje en cualquier tiempo y espacio. Así mismo, es importante que el modelo pedagógico sea coherente para adelantar un proceso de educación de alta calidad:

Dentro de una modalidad virtual hay muchas ventajas que permiten al estudiante tomarla como una alternativa de aprendizaje exitosa. Según Mendoza \& Galvis (1999, p. 34) se cuenta con un amplio listado de ventajas tanto para el tutor 
como para estudiantes, entre las más destacadas se encuentran: 1) Disminuir la sensación de aislamiento; 2) Incrementa flexibilidad y 3) Incrementa la variedad.

En este sentido, se resalta que dentro de un sistema educativo siempre se debe estar en constante evaluación y reflexión. El uso de las TIC debe favorecer el mejoramiento continuo dentro del entorno de aprendizaje para promover una educación de alta calidad, para ésto existen distintos instrumentos de aplicación de forma cualitativa y cuantitativa que permite fomentar la crítica constructiva en mejoramiento de los cursos en esta modalidad. Así mismo, se muestra la importancia de una educación superior en modalidad virtual donde el objetivo es brindar conocimiento de manera eficaz y pertinente en una cobertura total.

La evaluación en Ambientes Virtuales de Aprendizaje.

En la modalidad virtual es indispensable plantear una serie de pautas con el fin de desarrollar un sistema educativo de calidad. Para la creación de un ambiente virtual de aprendizaje es necesario tener en cuenta los siguientes elementos: Análisis, diseńo, desarrollo y evaluación, detrás de cada una de estas herramientas se plantean dinámicas que conlleven al éxito de aprendizaje en el estudiante. Para este tipo de evaluación constante se han venido creando instrumentos que permitan aplicarlos de forma continua en los cursos e ir reflexionando acerca de los cambios y dinámicas que deben configurarse en los cursos a evaluar. Para un tipo de evaluación es pertinente una selección de áreas y subáreas, definición de estándares y definición de indicadores con el fin de adoptar un sistema de mejoramiento dentro de un curso virtual. Como lo plantea Rubio $(2005,2003)$ al referir que los estándares de calidad y sus indicadores, al estar incluidos dentro de un modelo, adoptan las características intrínsecas del curso a evaluar, como aquellas otras que contribuyen a su organización y gestión; y que de alguna manera puedan afectar a la percepción de los estudiantes. Además, menciona que los indicadores establecidos, si bien han pretendido ser representativos de la globalidad del contexto, es conveniente que cada institución se- leccione los que más se adapten a sus necesidades y a su realidad institucional.

\section{Cursos metodológicos}

El programa académico de artes visuales en modalidad abierta y a distancia de una universidad pública en Colombia tiene un diseño curricular por núcleos problémicos, con lo cual se busca integrar de manera coherente el medio, la mediación, los mediadores, las estrategias de aprendizaje y la propuesta de evaluación, que gracias al orden metodológico, integrado por un conjunto de procedimientos, estrategias y técnicas pedagógico-didácticas, viabilizadas por los recursos y herramientas propias de los ambientes virtuales de aprendizaje, hacen posible el ejercicio de determinadas prácticas profesionales, oficios u ocupaciones, así como el reconocimiento de problemáticas regionales y sus factibles soluciones, al igual que la identificación de las nuevas exigencias formativas (PAP Solidario-UNAD, 2011, p.16).

En relación con la tipología, en el programa de artes se ofertan tres tipos de cursos: teóricos, metodológicos (teórico/prácticos) y prácticos. Los cursos teóricos del programa responden al saber declarativo del conocimiento, es decir, aquellos conceptos, fundamentos y problemas propios del saber y el conocimiento disciplinar. Los cursos metodológicos enfatizan en el saber procedimental, muy pertinente para la estrategia metodológica del taller y requiere la aplicación de conocimientos a problemáticas prácticas en las que el estudiante debe potenciar la aplicación de los contenidos teóricos (saber-hacer). Los cursos prácticos (talleres), son aquellos en los cuales se puede desarrollar la producción y acción; se estructuran a partir de los procesos de creación e investigación propios de cada estudiante una vez cuenta con los conocimientos y la experticia que ha asumido de los cursos teóricos y prácticos. En coherencia con lo anterior, se determinaron como cursos metodológicos del programa de artes visuales incluidos en el estudio los siguientes: Dibujo, Fotografía artística y dibujo básico. 
Estrategia de evaluación para el mejoramiento continuo de cursos de un programa de Artes Visuales en modalidad virtual y a distancia: Estudio de caso

Contexto y alcances de la investigación mediada por tecnologías de la información y la comunicación para la evaluación de un programa académico.

Según Romero (2014) a finales de los años sesenta la aparición de Internet gestó transformaciones en nuestras sociedades, conformadas ahora, por sujetos y subjetividades hiper-conectadas; ciudadanos del mundo, en territorios delimitados y con nuevas necesidades de consumo tecnológico (p.28). Para Garcés, Ruiz \& Martínez (2014, p.219) las sociedades reconocen el impacto social de Internet. Las transformaciones en las dinámicas, formas de interpretar las realidades e inter-relaciones del individuo consigo mismo, los otros y el medio ambiente, ha puesto en la agenda de discusión el compromiso de la Universidad de hoy para afrontar el reto de la educación superior en las sociedades digitales y su cultura. Dentro del impacto de transformación, las TIC empiezan a jugar un papel fundamental en los procesos pedagógicos como un aporte a la construcción de saberes, para la solución de problemas y situaciones de un entorno o contexto determinado.

La Universidad, desde su responsabilidad social y sus funciones sustantivas, las cuales según la UNESCO son la docencia, la investigación y la extensión, la que ha permitido dar el salto entre la educación tradicional a la educación a distancia, reconociendo otros modelos de enseñanza-aprendizaje y conservando estándares de calidad, pertinencia, inclusión social, integralidad, internacionalización y movilidad (Estrada, Fernández, \& Zambrano, 2017). Desde la docencia y la extensión se amplía el diseño y oferta de formación en AVA y cursos MOOC (cursos en línea, masivos, abiertos y gratuitos) y desde la investigación se ha desarrollado nuevo conocimiento acerca de los Entornos Virtuales para la mediación de procesos de investigación universitaria. Como se menciona anteriormente la investigación es un factor importante para el desarrollo de nuevo conocimiento y de nuevos productos que puedan aportar a los distintos campos de acción que se requieran; En este caso se ha dado la exploración de temáticas sobre las humanidades que antes manejaban incógnitas, y ahora se puntualiza frente a la investigación mediadas por tecnologías que contribuyen a mejorar, fortalecer y puntualizar las prácticas en los investigadores de las ciencias sociales y humanidades. (Romero, 2014, p. 20).

En la actualidad no sólo la investigación si no la misma educación es un desafío constante para lograr un aprendizaje significativo y una evolución de la transmisión de información; Es allí donde las tecnologías, la virtualización y la misma educación a distancia, contribuye a la construcción de un desarrollo social, a través de espacios virtuales donde potencien el aprendizaje autónomo. Haciendo uso de las tecnologías a través de la búsqueda de información independiente que conlleve a la edificación de un conocimiento individual, mediado de manera simultánea con aprendizaje colaborativo para el desarrollo de habilidades en ambientes virtuales. Estos espacios generan ambientes de discusión y de una búsqueda de transformación en formas de enseñanza, para llegar a un punto final y es la construcción de conocimiento, es allí donde la tecnología para la educación es una necesidad para evolucionar. (Navarro, 2017, p. 38).

En este sentido, se ha desarrollado cómo en la evolución de dichas tecnologías se permite la transmisión y transformación de espacios como en este caso lo es la educación, y es allí donde las TIC han generado parámetros de evolución en educación dentro de ambientes virtuales de aprendizaje, para esto se resaltan tres aspectos importantes a tener en cuenta para la construcción de espacios virtuales donde se dará información; Primero se busca comunicar, y es donde hace mención a transmitir una información sobre una temática determinada, para en un segundo momento cooperar, en el cual se fomenta un trabajo colaborativo para la construcción colectiva de conocimiento y por último compartir, donde el mediador de procesos fomente el estimulo para reforzar el aprendizaje inicial. (Real, 2011, p.54).

Teniendo en cuenta lo anterior, se resalta cómo la investigación mediada por tecnologías permite el diseño de una estrategia de evaluación de cursos metodológicos de un programa de artes en ambientes virtuales de aprendizaje, concentrando la atención del presente texto, por un lado, en el diseño de cursos metodológicos, sus estrategias 
pedagógicas y didácticas como campo de estudio; y por otro, Internet y las TIC como mediador de procesos de investigación colaborativa.

Desde el componente estratégico de investigación, del programa académico en Artes visuales, se estructura desde la formulación de la línea de investigación: Las artes en la era digital, cuya finalidad es desarrollar diversos parámetros investigativos dentro de las artes para el abordaje de los contextos contemporáneos, con el propósito de lograr la comprensión del uso y tratamiento de la producción de obra en los diferentes entornos sociales y culturales.

Considerando estos aspectos, en este estudio se tuvo como objetivo general: Desarrollar una estrategia de evaluación para el mejoramiento continuo de cuatro cursos metodológicos del programa de artes visuales en ambientes virtuales de aprendizaje: Dibujo, Fotografía y Diseño básico. Y como objetivos específicos: i. Formular los parámetros evaluativos para el mejoramiento continuo de los cursos a trabajar. ii. Identificar estrategias para el mejoramiento de los cursos del programa de Artes Visuales con los docentes y estudiantes. iii. Diseño de una herramienta para el mejoramiento continuo de los cursos dentro del programa.

\section{Materiales Y Métodos}

\section{Participantes}

En el año 2016, inicia el programa de artes visuales con una matricula de 50 estudiantes activos, para el siguiente año el número se incrementa a 278. La población de estudiantes, es heterogénea, de diferentes edades que oscilan entre los 16 a los 45 años y condiciones socio-económicas toda vez que es una universidad con un modelo pedagógico caracterizado por la inclusión social con presencia en 63 centros de educación abierta y a distancia distribuidos en todas las regiones de Colombia. Los participantes no presentan ninguna condición de discapacidad. La investigación cuenta con estudiantes del programa de artes distribuidos en los 63 municipios de Colombia como se muestra en la Tabla No. 2.
Tabla 2.

Ubicación geográfica de los estudiantes

\begin{tabular}{|c|c|c|c|}
\hline Cantidad & Región & Cantidad & Región \\
\hline 135 & Bogotá & 1 & La Dorada \\
\hline 16 & Medellín & 2 & La Plata \\
\hline 8 & Acacias & 1 & Málaga \\
\hline 5 & Barrancabermeja & 2 & Ocaña \\
\hline 6 & Barranquilla & 12 & Palmira \\
\hline 10 & Bucaramanga & 5 & Pasto \\
\hline 8 & Cali & 4 & Pitalito \\
\hline 3 & Cartagena & 1 & Sahagún \\
\hline 1 & Corozal & 1 & San José del Guaviare \\
\hline 3 & Cúcuta & 4 & Santa Martha \\
\hline 1 & Cumaral & 1 & Santander de Quilichao \\
\hline 6 & Dosquebradas & 2 & Sogamoso \\
\hline 5 & Duitama & 1 & Valle de Guamuez \\
\hline 10 & Facatativá & 1 & Tumaco \\
\hline 5 & Florencia & 3 & Valledupar \\
\hline 1 & Garagoa & 2 & Yopal \\
\hline 2 & Girardot & 5 & Zipaquirá \\
\hline \multirow[t]{2}{*}{5} & Ibagué & & \\
\hline & & Total & 278 \\
\hline
\end{tabular}

Análisis basado en información del SNIES, Ministerio de Educación Nacional, 2013

El muestreo fue de tipo voluntario, siguiendo la definición propuesta por Hernández, Fernández \& Baptista (2014, p.156) quien define la muestra de tipo voluntario como "...en la muestra voluntaria la selección de los participantes depende de circunstancias muy variadas. A esta clase de muestra también se le puede llamar autoseleccionada, ya que las personas se proponen como participantes en el estudio o responden a una invitación”.

A partir de la población universo antes descrita, se realiza una convocatoria para participación voluntaria en el proceso de evaluación de los cursos, remitiendo invitación a estudiantes inscritos en cursos metodológicos (teórico/prácticos) y de la cual se obtiene la siguiente distribución de participantes, según periodo académico de matrícula activa, lo que se muestra en la tabla 3. 
Estrategia de evaluación para el mejoramiento continuo de cursos de un programa de Artes Visuales en modalidad virtual y a distancia: Estudio de caso

Tabla 3.

Distribución de la muestra

\begin{tabular}{lc}
\hline $\begin{array}{l}\text { Periodo de la aplicación } \\
\text { (16 semanas) }\end{array}$ & $\begin{array}{c}\text { Muestra } \\
\text { (participantes) }\end{array}$ \\
\hline Enero - Junio 2016 & 39 \\
Enero - Junio 2017 & 20 \\
Agosto - Diciembre 2017 & 29 \\
\hline
\end{tabular}

Fuente: Elaboración propia (2017).

En relación con los docentes que dirigen los cursos metodológicos (teórico/prácticos) se propuso el desarrollo de un grupo focal. Se resalta la participación de cuatro (4) profesores, adultos jóvenes con edades entre los 25 y 35 ańos de edad, de los cuales tres (3) cuentan con un perfil de maestros en artes plásticas y visuales y uno (1) con perfil de especialista en fotografía. Además, cuentan con formación complementaria en el campo específico de docencia en Ambientes Virtuales de Aprendizaje (AVA).

\section{Instrumento}

Entendiendo el proceso de recolección de datos como un proceso sistemático que permita garantizar, por un lado, captar la información más determinante sobre la realidad, y por otro, ir chequeando la validez de las observaciones tentativas a partir de las distintas fuentes de observaciones o de las distintas fuentes para examinar una realidad (Bonilla-Castro \& Rodríguez-Sehk, 2005, p. 141). Desde este sentido, los instrumentos utilizados para la recolección de datos fueron principalmente dos:

1. Encuesta en línea: compuesta por 13 preguntas orientadas al abordaje de las siguientes categorías de análisis:

a. Expectativas frente al programa y metodología

a. Material didáctico

a. Acompañamiento docente

a. Nuevos recursos: Estrategia de comunicación.

El propósito del instrumento se concentro en la medición del alcance del aprendizaje de cursos metodológicos de artes visuales en metodología virtual. Para la aplicación de la encuesta se convocó a estudiantes de primera matrícula registrados en la base de datos del Programa de Artes Visuales inscritos en los cursos de Dibujo y Fotografía Artística. El diligenciamiento de la encuesta fue de carácter voluntario y anónimo aspectos comunicados en el encabezado del formulario en línea y parte del consentimiento informado.

La encuesta fue validada mediante una prueba piloto en la que se realizaron ajustes sobre las categorías analíticas de: a. Medios; b. Estrategias didácticas y c. Abordaje metodológico de los cursos.

2. Grupo Focal: Desde su ejercicio se constituyen en una entrevista grupal, guiada por una conversación colectiva en la cual los participantes exponen su experiencia, puntos de vista, percepciones y otros sobre un tema específico. Para su aplicación, se convocó al 100\% de los docentes de artes visuales que tenían el rol de directores de curso y tutores de cursos metodológicos.

Se realizó en dos sesiones con el propósito de indagar sobre los materiales e insumos, así como percepciones y expectativas en el proceso de diseño de cursos, teniendo en cuenta las actividades del curso y las acciones del programa para facilitar la realización de dichas actividades. El grupo focal para cada una de sus sesiones contó con la participación de 5 docentes y concentró su desarrollo sobre la indagación de las siguientes categorías analíticas: a. Diseño de curso: tecno-pedagógico y disciplinar; b. Didáctica y c. Acompañamiento docente. (Ver Tabla 4).

Es preciso resaltar que para las dos técnicas de recolección de datos antes mencionadas se tiene como referente central que en las investigaciones cualitativas prima la interpretación subjetiva propuesta por Max Weber (Castillo-Guzmán, 2003) lo que no impide la objetividad de sus resultados en términos de la validez de la significación, es decir de la capacidad de verificar los datos en virtud de que son realmente el resultado de una comprensión por parte del sujeto, colocando las interpretaciones en el contexto de la "realidad vivida por éstos y en la óptica de una comprensión más válida del mundo social” (Poutois \& Desnet, 
1992, p.68, citado por Castillo-Guzmán, 2003).

Tabla 4.

Instrumentos y alcance.

\begin{tabular}{ll}
\hline Tipo de Instrumento & \multicolumn{1}{c}{ Alcance } \\
\hline $\begin{array}{l}\text { Guía de preguntas } \\
\text { para Grupo Focal }\end{array}$ & $\begin{array}{l}\text { Acercamiento a la vivencia mis- } \\
\text { ma del estudiante de artes en la } \\
\text { modalidad virtual, permitió conocer } \\
\text { de primera voz la experiencia de un } \\
\text { artista en formación desde la modali- } \\
\text { dad a distancia. }\end{array}$ \\
\hline Encuesta en línea & $\begin{array}{l}\text { Identificación del alcance del apren- } \\
\text { dizaje de cursos metodológicos de } \\
\text { artes visuales en metodología virtual: } \\
\text { 1. Expectativas frente al programa y } \\
\text { metodología }\end{array}$ \\
& $\begin{array}{l}\text { 2. Material didáctico } \\
\text { 3. Acompañamiento docente } \\
\text { 4. Nuevos recursos: Estrategia de } \\
\text { comunicación. }\end{array}$ \\
\hline
\end{tabular}

Fuente: Elaboración propia, 2017

Tipo y diseño de estudio

La investigación se desarrolló desde un diseño metodológico cualitativo, tipo estudio de caso, según Hernández, Fernández \& Baptista (2014) caracterizado por: i. Se realiza en ámbitos naturales de los participantes o unidades de análisis. ii. Las variables no están controladas ni manipuladas. iii. Los significados se toman de los propios participantes. iv. Los datos no se reducen únicamente a valores numéricos.

El procedimiento para el desarrollo metodológico del estudio permite la siguiente estructura por fases señaladas en la figura 2:

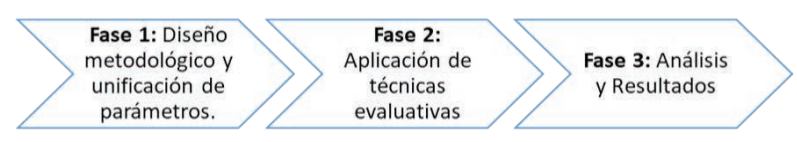

Figura 2. Fases del estudio

Fuente: Elaboración propia, 2018

Mediante el desarrollo sistemático de cada una de las fases se establecen tres categorías unificadas, su descriptor y código (Tabla 5), para el análisis de los resultados consolidados que permitieron la construcción de las herramientas de evaluación de los cursos metodológicos del programa.
Tabla 5

Categorías, descriptor y código

\begin{tabular}{lll}
\hline Categoría & Descripción & Código \\
\hline $\begin{array}{l}\text { Expectativas } \\
\text { frente al programa } \\
\text { y metodología }\end{array}$ & $\begin{array}{l}\text { Se expresa el cumplimiento } \\
\text { o no de las expectativas del } \\
\text { estudiante frente a los cursos } \\
\text { metodológicos. }\end{array}$ & \\
\hline Acompañamiento & Se expresa la favorabilidad o & AD \\
docente & $\begin{array}{l}\text { no de la calidad del acom- } \\
\text { pañamiento realizado por }\end{array}$ & \\
& $\begin{array}{l}\text { los docentes de los cursos } \\
\text { metodológicos. }\end{array}$ & \\
\hline Nuevos recursos: & $\begin{array}{l}\text { Se expresa participación o } \\
\text { no de los estudiantes en la }\end{array}$ & \\
comunicación & estrategia de comunicación del & \\
& programa de artes visuales - & \\
& UNAD. \\
\hline
\end{tabular}

Fuente: Elaboración propia (2017)

Posterior, se realiza el análisis cualitativo de los datos a partir de la codificación y categorización lo cual permitió establecer las recurrencias y omisiones y el conocimiento sobre la percepción, expectativas y necesidades de estudiantes y docentes frente al proceso de enseñanza-aprendizaje de las artes visuales en modalidad virtual y a distancia.

\section{Resultados}

Los hallazgos encontrados en la presente investigación se concentran en:

\section{Expectativas frente al programa y metodología:} El 87\% de los estudiantes nuevos consideran que la metodología es adecuada para el aprendizaje en cursos metodológicos de artes visuales.
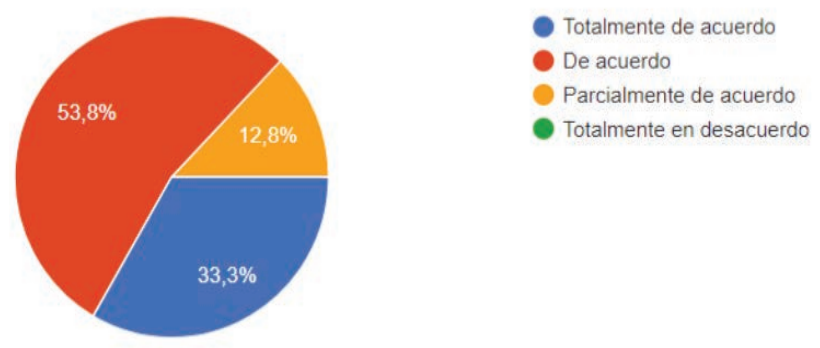

Figura 3. Fases del estudio

Fuente: Elaboración propia, 2018.

Así mismo, se han cumplido con las expectativas al momento de inscribirse al programa; Dentro 
Estrategia de evaluación para el mejoramiento continuo de cursos de un programa de Artes Visuales en modalidad virtual y a distancia: Estudio de caso

de esta categoría los estudiantes manifiestan que uno de los factores que más influye para tener éxito en los cursos es la respuesta y acompańamiento oportuno por parte del docente y así mismo con la categoría de material didáctico donde se busca que entre más apoyo de material tendrán una mejor comprensión de elaboración de productos para los cursos metodológicos. Teniendo en cuenta las expectativas dentro de la primera categoría, encontramos en la segunda categoría acerca de material didáctico que los estudiantes manifiestan que, aunque el producto visual es pertinente, la calidad del audio no permite potenciar cada uno de estos recursos para el aprendizaje de los cursos.

Acompańamiento docente: El 41\% de los estudiantes consideran que los docentes generan un acompańamiento pertinente para el desarrollo de los cursos metodológicos y que el curso se aborda de manera correcta permitiendo avanzar en el desarrollo de habilidades para las artes visuales, así mismo consideran que el material didáctico, y el desarrollo de los cursos ha promovido el aprendizaje significativo en cursos metodológicos de artes visuales, donde el vídeo permite que en su explicación paso a paso apliquen conceptos para culminar el proceso con educación de alta calidad.

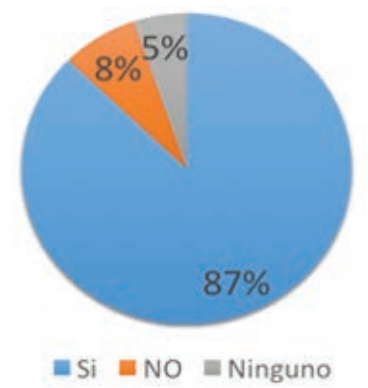

Figura 4. Comprensión de contenidos de Cursos Fuente: Elaboración propia, 2017
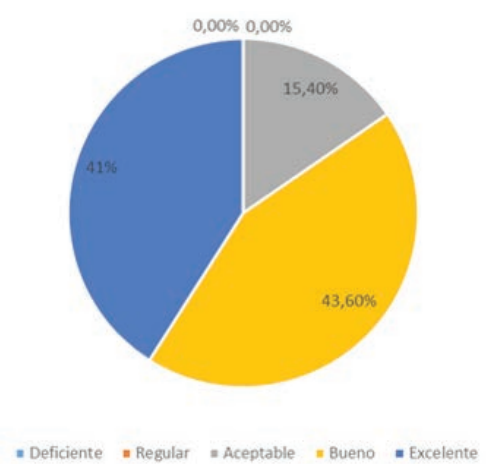

Figura 5. Calidad del acompañamiento docente Fuente: Elaboración propia, 2017

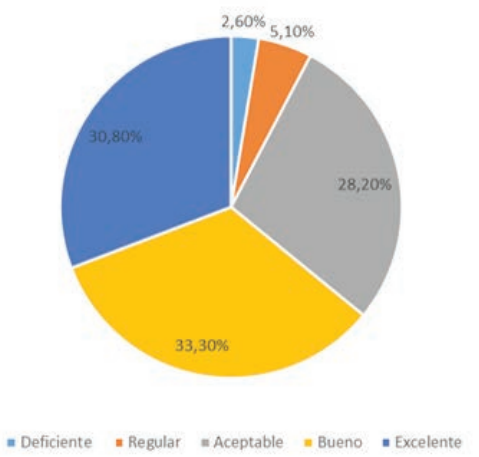

Figura 6. Comprensión de las actividades y contenidos Fuente: Elaboración propia, 2017

Así mismo, según la figura 5 y 6 se representa el $87 \%$ considera que los contenidos de los cursos metodológicos de artes visuales son comprensibles desde su contenido y el 30.8\% considera comprensible sus actividades. Sin embargo, estudiantes participantes expresan en un $28 \%$ una aceptable comprensión de las actividades en conjunto con un $15.4 \%$ quienes indican una aceptable calidad en el acompañamiento docente. Lo anterior, refleja la necesidad de replantear aspectos estructurales en el marco del diseño de cursos y recursos educativos digitales que faciliten con claridad e innovación los contenidos propios de los cursos teórico - metodológicos y del acompañamiento docente, desde los espacios propicios para ello como las mediaciones que permiten la realización de dicho proceso.

Nuevos recursos: estrategia de comunicación: Se exponen en la figura 7 y 8 que el $87 \%$ de los estudiantes percibe alta pertinencia temática en el espacio de vida académica denominada "Artes en Contexto" en contraste con un $41 \%$ que reporta no participar en el espacio. El programa de artes visuales desde la modalidad deberá promover y dar a conocer y comprender la importancia de estos recursos complementarios de actualización y vida académica universitaria que si bien en ocasiones no son in situ se desarrollan mediante transmisiones desde salas de web conferencia, desde la producción de programa de radio virtual de "Artes en Contexto" y abrir nuevos espacios de circulación y divulgación de productos derivados de los cursos teórico-metodológicos tales como revista de investigación formativa, evento académico de carácter nacional, redes académicas, grupo de investigación y semilleros de investigación. 
En complemento, articular desde lo curricular el Museo Universitario de Artes Digitales - MUNAD como pilar fundamental para todo proceso de divulgación de obra.

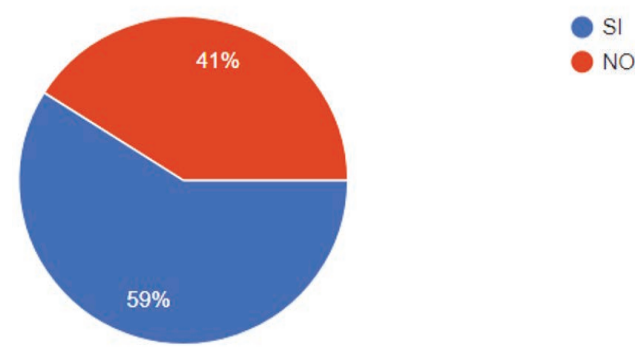

Figura 7. Participación en Artes en Contexto Fuente: Elaboración propia, 2017

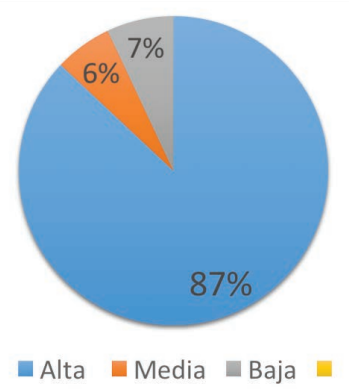

Figura 8. Pertinencia de las temáticas de Artes en Contexto Fuente: Elaboración propia, 2017

Como resultado del análisis, el equipo de docentes del programa académico diseña la siguiente herramienta de recolección e identificación de aspectos a mejorar en el proceso de diseño de cursos y recursos educativos digitales para la enseñanza de las artes visuales en ambientes virtuales de aprendizaje (Anexo1). Dentro de esta ficha de recolección se incluye una nueva categoría, la cual a partir del análisis de resultados se dispone para el mejoramiento de aspectos generales del programa desde su diseño curricular.

En consecuencia, desde la Coordinación Nacional del programa académico se dispone de la actualización del espacio de vida académica "Artes en Contexto" como estrategia de comunicación para la Ciencia, la Tecnología y la Innovación CteI (Anexo 2). Así como el diseño y puesta en marcha de la revista de investigación formativa Back Projection y el evento académico nacional MEDIÁTICA (Anexo 2) como primer encuentro de las artes digitales en Colombia que permitió aunar esfuerzos conjuntos entre 3 universidades colombianas para la creación de la Red Académica AVI - Arte, Virtualidad e Investigación. Estas acciones han permitido que los resultados obtenidos desde el proceso de investigación para la evaluación del programa redunden en decisiones estratégicas para la mejora continua y el acercamiento a altos estándares de calidad, así como en mejores recursos educativos digitales desde la pertinencia e innovación de los mismos.

\section{Discusión y Conclusiones}

La enseñanza de las artes en la modalidad virtual implica el desarrollo e implementación de una cultura de la evaluación y mejoramiento continuo de los recursos pedagógicos y didácticos que emplean los estudiantes para el desarrollo de diferentes actividades académicas en los cursos. La investigación permitió formular parámetros evaluativos a través de los instrumentos aplicados a docentes y estudiantes del programa de artes visuales. También ha permitido plantear el programa de Artes Visuales en modalidad virtual como objeto de investigación, implicando que la efectividad y posibilidades académicas de la modalidad se revisen a la luz de la evidencia de aplicación de instrumentos, resultados y análisis.

En este orden de ideas la investigación se constituye en un insumo para posteriores ejercicios de acreditación de alta calidad y registro calificado para la condición de investigación y mejoramiento curricular.

En coherencia con lo anterior, los instrumentos y la estrategia que se formuló en la presente investigación han permitido al comité curricular del programa tomar decisiones estratégicas de orden didáctico, pedagógico o comunicativo a partir de la información de los resultados y observaciones.

El impacto de la interpretación de los resultados suscita acciones de orden micro-curricular (cursos académicos) que redundan en ajustes permanentes para el mejoramiento continuo del programa, al permitir la apropiación de metodologías, en específico, de cursos como fotografía y dibujo. Toda vez que la calidad de los materiales didácticos y 
Estrategia de evaluación para el mejoramiento continuo de cursos de un programa de Artes Visuales en modalidad virtual y a distancia: Estudio de caso

del acompañamiento docente son un criterio esencial para el cumplimento de los objetivos de aprendizaje de los cursos en función.

Siguiendo el orden de lo expuesto desde autores tales como Ávila \& Bosco, 2001; Belloch, 2002; Mendoza, 1999 quienes concluyen que desde los procesos de enseñanza aprendizaje mediados por el uso de las TIC se posibilita el acceso a contenidos educativos en simultaneo con el acompańamiento activo por parte de los docentes, logrando que la experiencia, percepción y procesos de aprendizaje del estudiante de artes visuales se constituyan como eje fundamental para la formulación de procesos de investigación y mejoramiento en el diseño curricular. Es entonces como el plantear ejercicios constantes de indagación sobre las necesidades y requerimientos que tiene el estudiante en la modalidad virtual es impostergable en el desarrollo de las acciones estratégicas de mejoramiento. La experiencia del estudiante es crucial para el diseño de contenidos de los cursos futuros, la revisión permanente de los contenidos y estrategias garantiza que la efectividad de las acciones sobre el curso se pueda medir entre periodos.

Según lo señalado por la UNESCO (2004; 2016) la pertinencia de las dimensiones pedagógicas, didácticas, metodológicas y evaluativas dentro de un modelo pedagógico transformador, radica en la comprensión por afinar los procesos de aprendizaje a partir de las características, necesidades y transformaciones del entorno social, político y productivo que permea a los actores, como también trabajando en el fortalecimiento de una cultura educativa que garantice los derechos y deberes fundamentales desde la formación para el saber ser, el saber hacer, el saber aprender y el saber convivir.

En paralelo, la investigación en colaboración permite aunar esfuerzos conjuntos para desarrollar proyectos de investigación de alto impacto académico, social y educativo. Así como, otros escenarios que permitan el desarrollo de estrategias para la apropiación social del conocimiento, siendo las redes académicas y de investigación, las co-autorias nacionales e internacionales y los eventos científicos espacios propicios para el trabajo cola- borativo. Así mismo, la investigación permite la identificación de objetos de estudio relacionados con el programa académico en sí mismo, convirtiendo los cursos académicos, sus estrategias pedagógicas y didácticas e instrumentos en objetos de estudio orientados a la contribución significativa de aportes que permitan una real transformación pedagógica y que logren incidir en los paradigmas educativos propios de la educación virtual. Como lo refiere de Salinas (2012) que la agenda de investigación se oriente a lograr impacto real en las políticas educativas e institucionales, en el conocimiento de cómo ocurre el aprendizaje en estos escenarios, y como se da ese cambio en las prácticas educativas.

A futuros investigadores interesados en esta línea temática de abordar la enseńanza de cursos metodológicos (teórico-práctico) desde la modalidad virtual permitirá establecer metodologías que decanten en modelos de mejoramiento continuo aplicados para otros programas en la modalidad virtual y a distancia.

A modo de recomendaciones a futuro se puede incidir en que los retos y apuestas en los escenarios académicos e investigativos serán: 1 . La vida académica como escenario in situ de reconocimiento y apropiación de sentido de pertinencia institucional y para con el programa académico que se desarrolla, 2. Incrementar la producción de recursos educativos digitales a través de conferencias, encuentros presenciales estrategias B-learning, objetos virtuales de aprendizaje y otros con el fin de facilitar espacios para el fortalecimiento de la autonomía en el proceso de aprendizaje en ambientes virtuales. 3. Establecer estrategias pedagógicas que promuevan la interacción docente-estudiante a partir del uso constante de recursos educativos de tipo audiovisual. 4. Fortalecimiento de los escenarios de vida académica y universitaria que el programa dispone, específicamente en acciones para su divulgación entre la comunidad estudiantil.

Toda vez que estos escenarios brindan la posibilidad de complementar el conocimiento que se facilitan desde los cursos metodológicos del programa de artes visuales. Como diseñar herramientas metodológicas que posibiliten la evaluación de 
la apropiación de las TIC como mediadoras en procesos de formación profesional; promover la creación y consolidación de redes académicas y de investigación para el trabajo colaborativo entre instituciones que aborden las prácticas artísticas con énfasis en la producción, circulación y exhibición de las artes digitales en Colombia y Latinoamérica.

\section{REFERENCIAS BIBLIOGRÁFICAS}

Ávila, P. \& Bosco, M. (2001). Ambientes Virtuales de Aprendizaje una Nueva Experiencia. Recuperado de: http:// investigacion.ilce.edu.mx/panel_control/doc/c37ambientes.PDF

Barbosa, S. (2017). Documento de trabajo: Arte y Escuela. Programa de Artes Visuales, UNAD. Recuperado de: http:// hemeroteca.unad.edu.co/index.php/wp/article/view/2107

Belloch, C. (2012). Entornos virtuales de aprendizaje. Universidad de Valencia. España. Recuperado de https://www. uv.es/bellochc/pedagogia/EVA3.pdf

Bonilla-Castro, E. \& Roriguez-Sehk, P. (2005) Más allá del dilema de los métodos: La investigación en ciencias sociales. Uniandes, Santafé de Bogotá, Colombia.

Castillo-Guzmán, E. (2003) Lo científico de la investigación cualitativaviejos dilemas, nuevas posturas. Revista Nómadas, (18), p. 46-53. Recuperado de: https://dialnet.unirioja.es/ servlet/articulo?codigo $=3992040$

Estrada, O., Fernández, F. A., Zambrano, J., Mario \& L., Fuentes, R. (2017) El entorno virtual para la investigación científica y sus dimensiones. Revista Didasc@lia: Didáctica y Educación, 8, (1).

Garcés, M., Ruiz, R \& Matrínez, D. (2014). Transformación pedagógica mediada por tecnologías de la información y la comunicación. Colombia. Recuperado de https://dialnet.unirioja.es/descarga/articulo/5104968.pdf

Hernández, R., Fernández, C. \& Baptista, M. (2014) Metodología de la Investigación. Editorial Mc Graw Hill. México.

Mendoza, P. \& Galvis, A. (1999). Ambientes virtuales de aprendizaje: Una Metodología para su creación. Colombia. Uniandes, Informática Educativa.

Ministerio de Comunicaciones (2008). Plan Nacional de Tecnologías de la Información y la Comunicación. Recuperado de: http://eduteka.icesi.edu.co/pdfdir/ColombiaPlanNacionalTIC.pdf

Navarro, R. (2017). Educación mediada por tecnología: aprendizaje, innovación y prospectiva. Revista Iberoamericana, Universidad de Alcalá. Recuperado de https://www.researchgate.net/publication/319381407_Educacion_mediada_por_tecnologia_Aprendizaje_innovacion_y_prospectiva
Real, J. (2011). Modelos educativos y TIC. Recuperado de http://www.slideshare.net/realjulio/modelos-educativos-enel-uso-de-las-tic

Romero, E. (2014). Ciencias Sociales y Humanidades Digitales: una visión introductoria. Romero, E. \& Sánchez. M. (edit.), Ciencias Sociales y Humanidades Digitales: técnicas, herramientas y experiencias de e-Research e investigación en colaboración. Argentina: Editorial Sociedad Latina de Comunicación Social.

Rubio, M. J. (2003). Memoria: "Centro Virtual para el Desarrollo de Estándares de Calidad para la Educación Superior a Distancia en América Latina y el Caribe". Universidad Técnica Particular de Loja.

Rubio, M. J. (2005). Proceso de Autoevaluación de los Programas de Educación a Distancia basa en el Proyecto "Centro Virtual para el Desarrollo de Estándares de Calidad para la Educación Superior a Distancia en América Latina y el Caribe". Cuadernillos del trabajo del 1 al 9 y documento introductorio. Universidad Técnica Particular de Loja. Ecuador.

Salinas, J. (2012) La investigación ante los desafíos de los escenarios de aprendizaje futuros. Revista RED, 32, p.p. 1-23. Recuperado de: https://www.um.es/ead/red/32/salinas.pdf

SNIES-Sistema Nacional de Información de la Educación Superior (2013). Ministerio de Educación, Colombia. Reporte SNIES 2013, Módulo en Excel.

PAP Solidario-UNAD (2011). Plan de Desarrollo 20112015: "Educación para todos con calidad Global". Universidad Nacional Abierta y a Distancia (UNAD). Bogotá D.C. Colombia.

UNESCO (2016). Competencias y Estándares TIC: desde la dimensión pedagógica. Competencias y estándares TIC desde la dimensión pedagógica: Una perspectiva desde los niveles de apropiación de las TIC en la práctica educativa docente. Ed: Universidad Javeriana, Bogotá, Colombia. Recuperado de: http://www.unesco.org/new/fileadmin/MULTIMEDIA/FIELD/Santiago/pdf/Competencias-estandares-TIC.pdf

UNESCO (2004). Las TICs en la formación docente. Guía de planificación. París: Organización de las Naciones Unidas para la Educación, la Ciencia y la Cultura.

Zapata-Ros, M. (2015). Teorías y modelos sobre el aprendizaje en entornos conectados y ubicuos. Bases para un nuevo modelo teórico a partir de una visión crítica del "conectivismo". Teoría de la Educación. Educación y Cultura en la Sociedad de la Información, 16(1), 69-102. Recuperado de http://campus.usal.es/-revistas_trabajo/index.php/revistatesi/article/view/eks201516169102/12985 